\title{
A GLOBAL LOJASIEWICZ INEQUALITY FOR ALGEBRAIC VARIETIES
}

\author{
SHANYU JI, JÁNOS KOLLÁR AND BERNARD SHIFFMAN
}

\begin{abstract}
Let $X$ be the locus of common zeros of polynomials $f_{1}, \ldots, f_{k}$ in $n$ complex variables. A global upper bound for the distance to $X$ is given in the form of a Lojasiewicz inequality. The exponent in this inequality is bounded by $d^{\min (n, k)}$ where $d=\max \left(3, \operatorname{deg} f_{i}\right)$. The estimates are also valid over an algebraically closed field of any characteristic.
\end{abstract}

Let $f$ be a real analytic function on $\mathbb{R}^{n}$ and let $Z=\left\{x \in \mathbb{R}^{n} \mid f(x)=0\right\}$. Let $\operatorname{dist}(x, Z)=\inf _{z \in Z}\|x-z\|$ where $\|\cdot\|$ denotes the Euclidean norm. For any $x \in \mathbb{R}^{n}$ one expects to be able to compare $\operatorname{dist}(x, Z)$ and $f(x)$. This is done by the Lojasiewicz inequality:

1. Theorem (Lojasiewicz [L1, Theorem 17], see also [M, Theorem 4.1]). With the above notation, for any compact set $K$ there are positive constants $C$ and $\alpha$ such that $\operatorname{dist}(x, Z)^{\alpha} \leq C \cdot|f(x)|$ for every $x \in K$.

In general $\alpha$ can be large. For example [L2, p. 85] if $f(x, y)=y^{2 m}+$ $\left(y-x^{m}\right)^{2}$ then $\alpha \geq 2 m^{2}$. Also, it is not clear how $\alpha$ depends on $f$.

If $Z \subset \mathbb{C}^{n}$ is defined by complex analytic equations $f_{1}=\cdots=f_{k}=0$, then viewed as a real analytic set $Z \subset \mathbb{C}^{n} \cong \mathbb{R}^{2 n}$ it is defined by $f=0$ where $f=\left|f_{1}\right|^{2}+\cdots+\left|f_{k}\right|^{2}$ is a real analytic function. Thus the Lojasiewicz inequality applies to complex analytic or algebraic sets too.

If the defining equations $f_{i}$ are polynomials, one would like to estimate the exponent $\alpha$ in terms of the degrees of the polynomials. Recently Brownawell [B1] (see also [BY, Section 3]) proved such a bound for polynomials over the complex field. A polynomial has more complex zeros than real ones; thus one expects the complex case to be easier. In fact Brownawell's methods (and also ours) do not apply in the real case.

2. Theorem (Brownawell [B1]). Let $f_{1}, \ldots, f_{k} \in \mathbb{C}\left[z_{1}, \ldots, z_{n}\right]$ and $D=$ $\max \operatorname{deg} f_{i}$. Let $Z=\left\{z \in \mathbb{C}^{n} \mid f_{1}(z)=\cdots=f_{k}(z)=0\right\}$. Then there is a constant $C>0$ such that

$$
\left(\frac{\min (\operatorname{dist}(z, Z), 1)}{1+\|z\|^{2}}\right)^{(n+1)^{2} D^{\min (n, k)}} \leq C \cdot \max _{i}\left|f_{i}(z)\right|,
$$

where $\|z\|^{2}=\left|z_{1}\right|^{2}+\cdots+\left|z_{n}\right|^{2}$.

Received by the editors March 22, 1990.

1980 Mathematics Subject Classification (1985 Revision). Primary 14M10, 32C99; Secondary 32B99. 
The aim of this paper is to find the best possible exponent in terms of the degrees of the polynomials $f_{i}$. We need the following notation:

3. Notation. Given natural numbers $n \geq 2$ and $d_{1} \geq \cdots \geq d_{k}$ let

$$
B\left(n, d_{1}, \ldots, d_{k}\right)= \begin{cases}d_{1} \cdots d_{k} & \text { if } k \leq n \\ d_{1} \cdots d_{n-1} \cdot d_{k} & \text { if } k>n .\end{cases}
$$

For technical reasons related to the proofs in [B2] and [K] we also define

$$
\bar{B}\left(n, d_{1}, \ldots, d_{k}\right)=\left(\frac{3}{2}\right)^{j} B\left(n, d_{1}, \ldots, d_{k}\right)+\theta,
$$

where $j=\#\left\{i<\min (k, n)-1 \mid d_{i}=2\right\}$ and

$$
\theta= \begin{cases}1 & \text { if } k>n \text { and } d_{n-1}=2, \\ 0 & \text { otherwise. }\end{cases}
$$

We extend the above notation to any sequence $d_{1}, \ldots, d_{k}$ by first ordering it and then applying the above definitions.

4. Definition. Let $K$ be an algebraically closed field. By an absolute value we mean a valuation ||$: K \rightarrow[0, \infty)$ which satisfies the triangle inequality (and which can be Archimedean or not). Any basis of $K^{n}$ leads to a norm

$$
\left\|\left(x_{1}, \ldots, x_{n}\right)\right\|=\sqrt{\left|x_{1}\right|^{2}+\cdots+\left|x_{n}\right|^{2}} .
$$

If $V \subset K^{n}$ then we define

$$
\operatorname{dist}(x, V)=\inf _{y \in V}\|x-y\| .
$$

The following is our main result:

5. Theorem (Lojasiewicz-type inequality). Let $K$ be an algebraically closed field (any characteristic) and let $\|$ be an absolute value as in (4). Let $f_{1}, \ldots, f_{k} \in$ $K\left[x_{1}, \ldots, x_{n}\right]$ be polynomials and let $d_{i}=\operatorname{deg} f_{i}$. Assume that $n \geq 2$. Let $V=V\left(f_{1}, \ldots, f_{k}\right) \subset K^{n}$ be the common zero set of these polynomials. Assume that $V$ is nonempty.

Then there is a positive integer $m \leq \bar{B}\left(n, d_{1}, \ldots, d_{k}\right)$ and a constant $C>0$ (both depending on the $f_{i}$ ) such that

$$
\operatorname{dist}(x, V)^{m} \leq C \cdot \max _{i}\left\{\left|f_{i}(x)\right|\right\} \cdot(1+\|x\|)^{\bar{B}\left(n, d_{1}, \ldots, d_{k}\right)}
$$

holds for all $x \in K^{n}$.

Since $\operatorname{dist}(z, Z) \leq C^{\prime} \cdot(1+\|z\|)$ holds for some $C^{\prime}>0$, (5) implies the following improvement of Brownawell's result:

6. Corollary. Let $f_{1}, \ldots, f_{k} \in \mathbb{C}\left[z_{1}, \ldots, z_{n}\right]$ and let $d_{i}=\operatorname{deg} f_{i}$. Let $Z \subset \mathbb{C}^{n}$ be the common zero set of these polynomials. Then there is a constant $C>0$ such that

$$
\begin{gathered}
\left(\frac{\min (\operatorname{dist}(z, Z), 1)}{1+\|z\|}\right)^{\bar{B}\left(n, d_{1}, \ldots, d_{k}\right)} \leq C \cdot \max _{i}\left|f_{i}(z)\right| \text { and } \\
\left(\frac{\operatorname{dist}(z, Z)}{1+\|z\|^{2}}\right)^{\bar{B}\left(n, d_{1}, \ldots, d_{k}\right)} \leq C \cdot \max _{i}\left|f_{i}(z)\right|
\end{gathered}
$$

holds for all $z \in \mathbb{C}^{n}$. 
The proof of (5) will rest on Brownawell's version [B2] of the effective Nullstellensatz $[\mathrm{K}]$ :

7. Theorem [B2, Main Proposition]. Let $K$ be a field and let $\bar{f}_{1}, \ldots, \bar{f}_{k} \in$ $K\left[x_{0}, \ldots, x_{n}\right]$ be homogeneous polynomials of degree $d_{1}, \ldots, d_{k}$ respectively. Assume that $n \geq 2$.

Then there are prime ideals $P_{1}, \ldots, P_{s}$ containing $\left(\bar{f}_{1}, \ldots, \bar{f}_{k}\right)$ and there are natural numbers $e_{1}, \ldots, e_{s}$ such that

$$
\begin{aligned}
& \prod_{i=1}^{s} P_{i}^{e_{i}} \subset\left(\bar{f}_{1}, \ldots, \bar{f}_{k}\right) \quad \text { and } \\
& \sum_{i=1}^{s} e_{i} \cdot \operatorname{deg} P_{i} \leq \bar{B}\left(n, d_{1}, \ldots, d_{k}\right) .
\end{aligned}
$$

The "analytic" part of the proof of (5) is based on the following lemma in which we use the notation of (4)

8. Lemma. Let $Z \subset K^{n}$ be an irreducible subvariety of dimension $k$ and degree $d$. Then there are finitely many polynomials $g_{i}$ of degree at most $d$ vanishing on $Z$ and a constant $C$ such that

$$
\operatorname{dist}(x, Z)^{d} \leq C \cdot \max _{i}\left\{\left|g_{i}(x)\right|\right\} .
$$

Proof. Fix a generic projection $\Pi: K^{n} \rightarrow L$ where $L$ is a $k$ dimensional linear subspace. The restriction $\pi: Z \rightarrow L$ is finite of degree $d$ and surjective. For any given $x \in K^{n}$ let

$$
\operatorname{dist}_{\Pi}(x, Z)=\min _{y \in \pi^{-1}(\Pi(x))}\|x-y\| .
$$

It is clear that

$$
\operatorname{dist}(x, Z) \leq \operatorname{dist}_{\Pi}(x, Z) .
$$

Therefore it is sufficient to prove that

$$
\operatorname{dist}_{\Pi}(x, Z)^{d} \leq C \cdot \max _{i}\left\{\left|g_{i}(x)\right|\right\} .
$$

If $\operatorname{dim} Z=n-1$ then $Z$ is defined by a single polynomial $g \in K\left[x_{1}, \ldots, x_{n}\right]$ of degree $d$. Since a different basis gives a norm which is bounded by constant multiples of the first norm from below and above, we may choose coordinates such that $\Pi\left(x_{1}, \ldots, x_{n}\right)=\left(x_{1}, \ldots, x_{n-1}\right)$. Then $g=c x_{n}^{d}+\cdots$ where $c \neq 0$. Let $y_{1}, \ldots, y_{d} \in Z$ be the preimages of $\Pi(x)$ under $\pi$ (with multiplicities). Then

$$
\operatorname{dist}(x, Z)^{d} \leq \prod_{i=1}^{d} \operatorname{dist}\left(x, y_{i}\right)=\left|c^{-1} g(x)\right|=\left|c^{-1}\right| \cdot|g(x)| .
$$

Thus $C=\left|c^{-1}\right|$ is the desired constant.

We prove (9) by induction on the codimension. Suppose $\operatorname{codim} Z>1$; fix a hyperplane $H \supset L$ and $d+1$ generic lines $A_{i} \subset \operatorname{ker} \Pi$. Let $p_{i}: K^{n} \rightarrow H$ be the projections with kernel $A_{i}$. Let $Z_{i}=p_{i}(Z) \subset H$ be the images. By assumption (9) is true for the $Z_{i}$. 
It suffices to show that there is a constant $C^{\prime}$ such that

$$
\operatorname{dist}_{\Pi}(x, Z) \leq C^{\prime} \cdot \max _{i}\left\{\operatorname{dist}_{\Pi}\left(p_{i}(x), Z_{i}\right)\right\} .
$$

To see this define the $\varepsilon$-neighborhood of $A_{i}$ as

$$
U_{\varepsilon}\left(A_{i}\right) \stackrel{\text { def }}{=}\left\{x \in K^{n} \mid x=x_{a}+x_{h} ; x_{a} \in A_{i} ; x_{h} \in H \text { and }\left\|x_{h}\right\|<\varepsilon \cdot\left\|x_{a}\right\|\right\} .
$$

Note that if $x \notin U_{\varepsilon}\left(A_{i}\right)$ then $\|x\| \leq\left(1+\varepsilon^{-1}\right)\left\|p_{i}(x)\right\|$. Now choose $\varepsilon$ so that the $\varepsilon$-neighborhoods of the $A_{i}$ do not intersect each other.

Let $\pi^{-1}(\Pi(x))=\left\{y_{1}, \ldots, y_{p}\right\}$ (as sets); $p \leq d$. There are $(d+1)$ lines $A_{i}$ and at most $d$ points $x-y_{i}$. Thus there is an index $j$ (depending on $x$ ) such that

$$
\left\{x-y_{1}, \ldots, x-y_{p}\right\} \cap U_{\varepsilon}\left(A_{j}\right)=\varnothing .
$$

This implies that

$$
\operatorname{dist}_{\Pi}(x, Z) \leq\left(1+\varepsilon^{-1}\right) \max _{j}\left\{\operatorname{dist}_{\Pi}\left(p_{j}(x), Z_{j}\right)\right\} .
$$

10. Remark. It follows from the above proof that for a nonconstant polynomial $f \in K\left[x_{1}, \ldots, x_{n}\right]$

$$
\operatorname{dist}(x, V(f))^{\operatorname{deg} f} \leq C \cdot|f(x)| .
$$

Now we can prove (5).

We introduce a new variable $x_{0}$ and homogenize the polynomials $f_{1}, \ldots, f_{k}$ to get $\bar{f}_{1}, \ldots, \bar{f}_{k}$. Let $P_{1}, \ldots, P_{s}$ be the prime ideals in (7). Assume that they are indexed such that $x_{0} \in P_{r+1} \cap \cdots \cap P_{s}$ and $x_{0} \notin P_{1} \cup \cdots \cup P_{r}$. Let $Z_{1}, \ldots, Z_{r}$ be the affine varieties in $K^{n}$ corresponding to $P_{1}, \ldots, P_{r}$. Then $V=Z_{1} \cup \cdots \cup Z_{r}$.

By $(8)$ for each $Z_{i}$ we can find a finite collection of polynomials $\left\{g_{i, j}\right\}$ of degree at most $z_{i}:=\operatorname{deg} Z_{i}=\operatorname{deg} P_{i}$ and a positive constant $C_{i}$ such that $g_{i j}$ vanishes on $Z_{i}$ for each $j$ and

$$
\operatorname{dist}\left(x, Z_{i}\right)^{z_{i}} \leq C_{i} \cdot \max _{j}\left\{\left|g_{i, j}(x)\right|\right\} .
$$

Let $e_{1}, \ldots, e_{s}$ be as in (7). Then

$$
\begin{aligned}
\operatorname{dist}(x, V)^{z_{1} e_{1}+\cdots+z_{r} e_{r}} & \leq \prod_{i=1}^{r} \operatorname{dist}\left(x, Z_{i}\right)^{z_{i} e_{i}} \\
& \leq \prod_{i=1}^{r} C_{i}^{e_{i}} \prod_{i=1}^{r} \max _{j}\left\{\left|g_{i, j}(x)\right|^{e_{i}}\right\} \\
& \leq C^{\prime} \cdot \max _{j_{1}, \ldots, j_{r}}\left\{\left|\prod_{i=1}^{r} g_{i, j_{i}}(x)^{e_{i}}\right|\right\} .
\end{aligned}
$$

Thus we need to understand the polynomials

$$
\prod_{i=1}^{r} g_{i, j_{i}}(x)^{e_{i}}
$$

By (7) we conclude that

$$
x_{0}^{e_{r+1}+\cdots+e_{s}} \prod_{i=1}^{r} g_{i, j_{i}}(x)^{e_{i}} \in\left(\bar{f}_{1}, \ldots, \bar{f}_{k}\right) .
$$


Since the degree of $g_{i, j_{i}}$ is at most $z_{i}$, by (7) the degree of the left-hand side in (12) is at most $\bar{B}\left(n, d_{1}, \ldots, d_{k}\right)$. Thus there are polynomials $G_{i, j_{1}, \ldots, j_{r}} \in$ $K\left[x_{1}, \ldots, x_{n}\right]$ of degree at most $\bar{B}\left(n, d_{1}, \ldots, d_{k}\right)-d_{i}$ such that

$$
\prod_{i=1}^{r} g_{i, j_{i}}(x)^{e_{i}}=\sum_{i=1}^{k} G_{i, j_{1}, \ldots, j_{r}} f_{i} .
$$

Note that if $h \in K\left[x_{1}, \ldots, x_{n}\right]$ has degree at most $q$ then there is a constant $C^{\prime \prime}$ such that

$$
|h(x)| \leq C^{\prime \prime} \cdot(1+\|x\|)^{q} .
$$

Thus for a suitable constant $C^{\prime \prime}$

$$
\left|G_{i, j_{1}, \ldots, j_{r}}(x)\right| \leq C^{\prime \prime} \cdot(1+\|x\|)^{\bar{B}\left(n, d_{1}, \ldots, d_{k}\right)-d_{i}},
$$

where $C^{\prime \prime}$ is independent of $i, j_{1}, \ldots, j_{r}$. Therefore by (11)

$$
\begin{aligned}
\operatorname{dist}(x, V)^{z_{1} e_{1}+\cdots+z_{r} e_{r}} & \leq C^{\prime} \cdot \max _{j_{1}, \ldots, j_{r}}\left\{\left|\sum_{i} G_{i, j_{1}, \ldots, j_{r}} f_{i}\right|\right\} \\
& \leq k C^{\prime} C^{\prime \prime} \cdot \max _{i}\left\{\left|f_{i}(x)\right|\right\} \cdot(1+\|x\|)^{\bar{B}\left(n, d_{1}, \ldots, d_{k}\right)} .
\end{aligned}
$$

Take $m=\sum_{i=1}^{r} z_{i} e_{i}$ and $C=k C^{\prime} C^{\prime \prime}$ to get (5).

Note that we proved in fact a slightly stronger statement:

$$
\operatorname{dist}(x, V)^{m} \leq C \cdot \max _{i}\left\{\left|f_{i}(x)\right| \cdot(1+\|x\|)^{\bar{B}\left(n, d_{1}, \ldots, d_{k}\right)-d_{i}}\right\} .
$$

In this form both the bound on $m$ and the exponents of $1+\|x\|$ are the best possible, provided that $d_{i} \neq 2$.

15. Example. This variant of an example given by Masser and Philippon (see [B1; K, 2.3]) shows that the upper bound on $m$ in (14) is sharp (for $d_{i} \neq 2$ ).

Let $f_{1}=x_{2}-x_{1}^{d_{1}}, f_{2}=x_{3}-x_{2}^{d_{2}}, \ldots, f_{n-1}=x_{n}-x_{n-1}^{d_{n-1}}, f_{n}=x_{n}^{d_{n}}$. Then $V\left(f_{1}, \ldots, f_{n}\right)=\{0\}$. Let

$$
x(t)=\left(t, t^{d_{1}}, t^{d_{1} d_{2}}, \ldots, t^{d_{1} d_{2} \cdots d_{n-1}}\right) .
$$

Then $\operatorname{dist}(x(t), 0) \approx|t|$ for small $|t|$ but

$$
\max \left|f_{i}(x(t))\right|=\left|f_{n}(x(t))\right|=|t|^{d_{1} d_{2} \cdots d_{n}} .
$$

16. Example. This example shows that in some cases the only value of $m$ that works in (5) is $m=1$.

In $K[x, y]$ let $f_{1}=y, f_{2}=y(x-1)^{s}-x$ where $s \geq 2$. Then $\bar{B}(2,1, s+1)=$ $s+1$ and $V\left(f_{1}, f_{2}\right)=\{0\}$. Consider the family of points $z(t)=\left(t, t(t-1)^{-s}\right)$. Then $f_{2}(z(t))=0$ and for large values of $|t|$ we have

$$
\operatorname{dist}(z(t),\{0\})=\|z(t)\| \approx|t| \text { and }\left|f_{1}(z(t))\right| \approx|t|^{1-s} .
$$

Thus

$$
\max _{i=1,2}\left\{\left|f_{i}(z(t))\right| \cdot(1+\| z(t)||)^{\bar{B}\left(n, d_{1}, d_{2}\right)-d_{i}}\right\}=\left|f_{1}(z(t))\right| \cdot(1+\| z(t)||)^{s} \approx|t| .
$$

Hence we must take $m=1$ in (5). 
This example also shows that the exponent 2 of $\|z\|$ in (6.2) cannot be made smaller if the degrees go to infinity.

17. Remark. One can interpret (5) as follows: Given a system of equations $f_{1}=\cdots=f_{k}=0$ over an algebraically closed field $K$, let $\left(x_{1}, \ldots, x_{n}\right)$ be an approximate solution. Then there is an actual solution near $\left(x_{1}, \ldots, x_{n}\right)$. From this point of view the assumption that $V$ be nonempty is very inconvenient. This form is especially interesting when the absolute value is nonarchimedean, e.g. when $K$ is the algebraic closure of a complete discrete valuation ring. However in this case one would like to prove a similar result without assuming that $K$ is algebraically closed, or even for equations over any complete local ring. Such results are known [A, Chapter 6] but the bounds are probably far from being optimal.

18. Remark. Let us take this opportunity to correct an error in [K]. In the formulation of [K, Proposition 1.10] the exponent should be the above $B\left(n, d_{1}, \ldots, d_{k}\right)$ instead of $N\left(n, d_{1}, \ldots, d_{k}\right)$. (See [K, Section 4].) These two functions are conjecturally equal but the equality is proved only if all the $d_{i}$ are different from $2[\mathrm{~K}, 1.9]$.

\section{ACKNOWLEDGMENT}

We wish to thank C. Berenstein, Y. Shimizu, A. Yger and S. Zucker for their helpful conversations about this work.

Partial financial support for the first author was provided by a University of Houston Research Initiation Grant. Partial financial support for the second author was provided by the NSF under grant numbers DMS-8707320 and DMS- 8946082 and by an A. P. Sloan Research Fellowship. Partial financial support for the third author was provided by the NSF under grant numbers DMS-8701808 and DMS-8901571.

\section{REFERENCES}

[A] M. Artin, Algebraic approximation of structures over complete local rings, Publ. Math. IHES 36 (1969), 23-58.

[BY] C. A. Berenstein and A. Yger, Bounds for the degrees in the division problem, Michigan Math. J. 37 (1990), 25-43.

[B1] W. D. Brownawell, Local diophantine Nullstellen inequalities, J. Amer. Math. Soc. 1 (1988), 311-322.

[B2] _ A prime power product version of the Nullstellensatz, Michigan Math. J. (to appear).

[K] J. Kollár, Sharp effective Nullstellensatz, J. Amer. Math. Soc. 1 (1988), 963-975.

[L1] S. Lojasiewicz, Sur le problème de la division, Studia Math 18 (1959), 87-136.

[L2] _ Ensembles semi-analytiques, IHES, Bures-sur-Yvette, 1965.

[M] B. Malgrange, Ideals of differentiable functions, Oxford Univ. Press, 1966.

Department of Mathematics, University of Houston, Houston, Texas 77204

Department of Mathematics, University of Utah, Salt lake City, Utah 84112

Department of Mathematics, The Johns Hopkins University, Baltimore, Maryland 21218 